\title{
Design of Linear Time Varying Flatness-Based Control for Single-Input Single-Output Systems
}

\author{
Marouen Sleimi \\ Research Laboratory in Automatic \\ Control - LA.R.A \\ University of Tunis EL Manar \\ National Engineering School of Tunis, \\ BP-36, le Bilvedere 1002 Tunis
}

\author{
Mohamed Ben Abdallah \\ Higher Institute of Technology \\ Study of Rades, \\ BP 172, 2098, Radès Médina,Tunisia
}

\author{
Mounir Ayadi \\ Research Laboratory in Automatic \\ Control - LA.R.A \\ University of Tunis EL Manar \\ National Engineering School of Tunis, \\ BP-36, le Bilvedere 1002 Tunis
}

\begin{abstract}
In this paper, the control of linear discrete-time Varying Single-Input Single-Output systems is tackled. By using flatness theory combined with a dead-beat observer, a two degree of freedom controller is designed with high performances in terms of trajectory tracking. The aim of this work is to avoid the choice of closed loop poles in linear discrete-time varying framework which build a very serious problem in system control. The effectiveness of this control law is highlighted by simulation results.
\end{abstract}

Keywords-Flatness theory; discrete-time systems; linear time varying; single-input single-output; dead-beat observer; two degree of freedom controller

\section{INTRODUCTION}

The theory of linear time-invariant systems gives a wide range of design methods and solutions to control problems including all kinds of techniques such as state feedback controllers and observers, Kalman filters, $H_{2}$ control and $H_{\infty}$ control. Since then, researchers have kept on making efforts to extend the previous systems control approaches to timevarying systems and sampled-data systems.

LTV (Linear Time Varying) systems are of a great interest because of the fact that time invariant nonlinear systems can be approximated by LTV systems around desired trajectories after there linearization. However, a fundamental part in the study of LTV systems is insured by the state transition matrix noted $\phi$, which can be computed as the sum of the PeanoBaker series. We can note here that not all arguments and assumptions used of time-invariant systems are useful in timevarying framework. To extend them, time-varying systems are examined carefully on their controllability and stability.

Moreover, many control design approaches use LTV systems, one of the most important way of systems control is SMC (Sliding mode control) which is very used in the case of dynamic uncertain systems [1]. Furthermore, we can evaluate Back Stepping method which used to aim the objective of stabilizing nonlinear dynamic systems leading to an LTV system after linearization [2]. Besides, we find fuzzy control operating nonlinear systems to make nonlinear controllers via the use of heuristic information [3]. Among these control design approaches, flatness-based control remains the most suitable method in trajectory tracking, then in the rest of the paper, we are interested to this kind of controller in the LTV case.
Previously, it is shown that flatness property considerably simplifies the 2DOF (Two Degree of Freedom) controllers design for continuous-time SISO (Single-Input Single-Output) systems for LTV framework [4], [5]. In these works, the main feature of this flatness approach for 2DOF controllers design, using flatness-based control and dead-beat observer, is to avoid the choice of closed loop poles and no need to solve diophantine equation any more. In this design the closed loop dynamics are related to the chosen tracking dynamics.

This approach was extended to discrete-time framework for LTI SISO [6], [7] and LTI MIMO (Multiple-Input MultipleOutput) systems [8], [9], [10]. This paper extend the previous approaches to deal with LTV flatness-based control in SISO discrete-time framework.

This paper is organized as follows: In Section II, some preliminaries are presented. Then in Section III, the definition of the canonical controllable form in discrete-time SISO LTV framework is given. Moreover, in Section IV, the new approach of control design is developed. In Section V, effectiveness of this control law is illustrated using an academic discrete-time SISO system.

In the following, we will develop the paper in a discretetime formulation, using the shift forward operator $q$ and the delay operator $q^{-1}$.

\section{PRELIMINARIES}

Introducing a given problem concerning LTV systems, start generally by results given by LTI techniques and trying to adapt it in the new context. Flatness-based control is introduced and developed by Fliess and co-researchers [11] and used by many authors, firstly in the LTI framework then in the LTV one.

This work deals with flatness-based control in LTV discrete-time framework. So to aim this objective a canonical controllable form must be built and is exploited in the proposed control law design.

\section{A. Canonical controllable form for LTV discrete-time SISO systems}

Canonical forms are widely used in control theory. In this section, the discrete-time LTV controllable canonical form is presented [12]. 

by:

Considering the following discrete-time LTV system given

$$
\left\{\begin{array}{c}
x_{k+1}=A_{k} x_{k}+B_{k} u_{k} \\
y_{k}=C_{k} x_{k}
\end{array}\right.
$$

where $x_{k}$ is a $n$ dimensional vector, $A_{k}$ is a $(n \times n)$ matrix, $B_{k}$ and $C_{k}$ are a $m$ dimensional vectors.

If the given system is uniformly $\mathrm{N}$-step controllable, then there exist a transformation applied to the state given by:

$$
\bar{Z}_{k}=\bar{T} x_{k}
$$

where $\bar{T}_{k}$ is a Lyapunov transformation matrix, presented in Appendix A, obeying the conditions below.

- $\bar{T}_{k}$ is defined each sample time.

- $\quad \bar{T}_{k}$ and $\bar{T}_{k+1}$ are bounded each sample time.

- There exist a constant $m$ where:

$$
0<m<\operatorname{det}\left(\bar{T}_{k}\right), \quad k>0
$$

This algorithm of controllable form leads to a new state vector equation written as following:

$$
\left\{\begin{array}{c}
Z_{k+1}=\bar{A}_{k} Z_{k}+\bar{B}_{k} \bar{u}_{k} \\
y_{k}=\bar{C}_{k} Z_{k}
\end{array}\right.
$$

where $\bar{A}_{k}, \bar{B}_{k}$ and $\bar{C}_{k}$ are given by the new canonical controllable form for LTV systems, such as:

$$
\bar{A}_{k}=\bar{T}_{k+1} A_{k} \bar{T}_{k}^{-1}
$$

$$
=\left[\begin{array}{ccccc}
0 & 1 & 0 & & 0 \\
\vdots & \ddots & \ddots & \ddots & \vdots \\
\vdots & & \ddots & \ddots & 0 \\
0 & \ldots & \ldots & 0 & 1 \\
-\gamma_{0}(k) & -\gamma_{1}(k+1) & \ldots & \ldots & -\gamma_{n-1}(k+n-1)
\end{array}\right]
$$

$$
\bar{B}_{k}=\bar{T}_{k+1} B_{k}=\left[\begin{array}{c}
0 \\
\vdots \\
\vdots \\
0 \\
1
\end{array}\right]
$$$$
\bar{C}_{k}=C_{k} \bar{T}_{k+1}=\left(\begin{array}{lll}
\alpha_{1 k} & \ldots & \alpha_{(n-1) k}
\end{array}\right)
$$

\section{B. Flatness Properties}

Flatness can parametrize, in a very simple way, the dynamic aspect of a given system based on highlighting some endogenous fundamental variables called flat outputs. In fact, the state, the input and the output using the flat output can be written as follows:

$$
x_{k}=f_{1}\left(z_{k}, \quad z_{k+1}, \quad \ldots, \quad z_{k+n-1}\right)^{T}
$$

and

$$
u_{k}=f_{2}\left(z_{k}, \quad z_{k+1}, \quad \ldots, \quad z_{k+m-1}\right)^{T}
$$

$$
y_{k}=g_{k}\left(x_{k}, u_{k}\right)^{T}
$$

As known, on the first hand, the state, the input vector and the output vector in continuous time framework are defined as a successive derivatives of the flat output, on the other hand, in discrete-time framework it is considered as a phase advance sequence of the flat output.

Marlait et al. [13] results confirm that discrete-time LTV system is the equivalent of a controllable LTI system and noting that flatness of a given one is strongly related to the uniform controllability. To build up flatness-based control it is necessary that the considered system is flat, so firstly its controllability [12], [14] must be checked out. If the system is uniformly $\mathrm{N}$-step controllable then we can apply the control law. Let's consider the plant given by (1) and the transformation given by (2).

Noting that $z_{k}$ the first element of the state vector $Z_{k}$, then from (3) it's clear that $Z_{k}$ can be written as [11]:

$$
Z_{k}=\left[\begin{array}{llll}
z_{k} & z_{k+1} & \ldots & z_{k+n-1}
\end{array}\right]^{T}
$$

where $z_{k}$ is the Bronovsky output.

The expression of the input $u_{k}$ is a function of $z_{k}$, and its forward terms:

$u_{k}=z_{k+n}+\gamma_{n-1}(k+n-1) z_{k+n-1}+\ldots+\gamma_{1}(k+1) z_{k+1}$

$$
+\gamma_{0}(k) z_{k}
$$

(5) The output $y_{k}$ which depends on $z_{k}$ is written as follows:

$$
y_{k}=\bar{C}_{k} Z_{k}=\sum_{i=0}^{n-1} \alpha_{i k} z_{k+i}
$$

$z_{k}$ can be considered as a flat output of the discrete LTV system. From the canonical form given previously flatnessbased control for SISO LTV systems will be developed in the next section.

\section{FlatNESS-BASED CONTROL FOR SISO LTV SYSTEMS}

\section{A. Flatness-based Control}

Following (11) the flatness-based control law is given by:

$$
u_{k}=z_{k+n}^{d}+\sum_{i=0}^{n-1} \kappa_{i}\left(z_{k+i}^{d}-z_{k+i}\right)+\sum_{i=0}^{n-1} \gamma_{i}(k+i) z_{k+i}
$$

$\kappa_{i}$ are derived from the following polynomial:

$$
K(q)=q^{n}+\sum_{i=0}^{n-1} \kappa_{i} q^{i}
$$


which must be shur [15], [16]. To build this control it is necessary to estimate the state vector $Z_{k}$ using an observer [17]. In this paper the dead-beat observer is used in the design strategy leading to a $2 \mathrm{DOF}$ controller. The structure of the flatness-based control is given by the following expression:

$$
u_{k}=K(q) z_{k}^{d}+\Lambda_{k} Z_{k}
$$

with:

$$
\Lambda_{k}=\left[\begin{array}{c}
\gamma_{0 k}-\kappa_{0} \\
\vdots \\
\gamma_{i(k+i)}-\kappa_{i}
\end{array}\right]
$$

The control law schema is shown in Fig. 1.

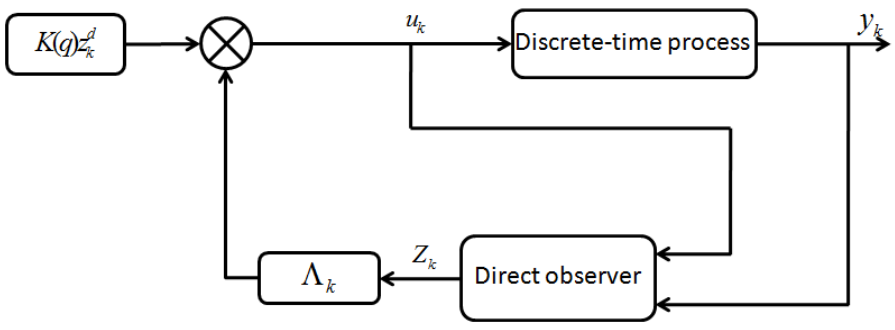

Fig. 1. Control law design.

\section{B. Dead-beat Discrete-time Varying Observer Design}

Successive iterations of the output equation in (3) to the order $(n-1)$ [18], give:

$$
\left(\begin{array}{c}
y_{k} \\
y_{k+1} \\
\vdots \\
y_{k+n-1}
\end{array}\right)=L_{k} Z_{k}+M_{k}\left(\begin{array}{c}
u_{k} \\
u_{k+1} \\
\vdots \\
u_{k+n-2}
\end{array}\right)
$$

with:

$$
L_{k}=\left(\begin{array}{c}
\bar{C}_{k} \\
\bar{C}_{(k+1)} \bar{A}_{k} \\
\bar{C}_{(k+2)} \bar{A}_{k+1} \bar{A}_{k} \\
\vdots \\
\bar{C}_{(k+n-1)} \bar{A}_{(k+n-2)} \ldots \bar{A}_{k}
\end{array}\right)
$$

and:

$$
M_{k}=\left[\begin{array}{cccc}
M_{1, k} & 0 & \cdots & 0 \\
M_{2, k} & M_{1, k+1} & \cdots & \vdots \\
M_{3, k} & M_{2, k+1} & \cdots & \vdots \\
\vdots & \vdots & \cdots & 0 \\
M_{n-1, k} & M_{n-2, k+1} & \cdots & M_{1, k+n-1}
\end{array}\right]
$$

where:

$$
M_{1, k}=\bar{C}_{k+1} \bar{B}_{k}
$$

and:

$$
M_{i, k}=\bar{C}_{k+i-1} \bar{A}_{k+i} \bar{B}_{k}
$$

Noting that:

$$
Y_{k}=\left(\begin{array}{ccc}
y_{k} & \ldots & y_{k+n-1}
\end{array}\right)^{T}
$$

and:

$$
U_{k}=\left(\begin{array}{lll}
u_{k} & \ldots & u_{k+n-2}
\end{array}\right)^{T}
$$

Equation (18) can be then written as:

$$
Y_{k}=L_{k} Z_{k}+M_{k} U_{k}
$$

Supposing that the system is uniformly N-step observable then the rank of $L_{k}$ is $n$ for all $k$. Noting that $L_{k}$ is the observability matrix, so:

$$
Z_{k}=L_{k}^{-1} Y_{k}-L_{k}^{-1} M_{k} U_{k}
$$

In a second hand:

$$
Z_{k}=q^{-1}\left(\bar{A}_{k} Z_{k}+\bar{B}_{k} u_{k}\right)
$$

After a second iteration:

$$
Z_{k}=q^{-1}\left(A_{k} q^{-1}\left(A_{k} Z_{k}+B_{k} u_{k}\right)+B_{k} u_{k}\right)
$$

The iteration of this expression to the order $l$ gives:

$$
Z_{k}=\phi_{l} q^{-l} Z_{k}+\sum_{i=1}^{l-1} \phi_{i} \bar{B}_{k-(i+1)} u_{k-(i+1)}+\bar{B}_{k-1} u_{k-1}
$$

with:

$$
\begin{gathered}
\phi_{l}=\prod_{j=1}^{l} \bar{A}_{k-j} \\
\phi_{0}=I_{n}
\end{gathered}
$$

$I$ is the identity matrix and $\phi_{l}$ is $(n \times n)$-dimensional matrices. By replacing $Z_{k}$ in the right side of (29) by the expression in (26), $Z_{k}$ can be written as follows:

$$
\begin{aligned}
& Z_{k}=\phi_{l} q^{-l}\left(L_{k}^{-1} Y_{k}-L_{k}^{-1} M_{k} U_{k}\right) \\
& +\sum_{i=1}^{l-1} \phi_{i} \bar{B}_{k-(i+1)} u_{k-(i+1)}+\bar{B}_{k-1} u_{k-1}
\end{aligned}
$$

then:

$$
\begin{aligned}
Z_{k}=\phi_{l} L_{k-l}^{-1}\left(\begin{array}{c}
y_{k-l} \\
\vdots \\
y_{k}
\end{array}\right)-\phi_{l} L_{k-l}^{-1} M_{k-l}\left(\begin{array}{c}
u_{k-l} \\
\vdots \\
u_{k+n-2-l}
\end{array}\right) \\
+\sum_{i=1}^{l-1} \phi_{i} B_{k-(i+1)} u_{k-(i+1)}+B_{k-1} u_{k-1}
\end{aligned}
$$

As $Z_{k}$ is written as:

$$
Z_{k}=\phi_{l} L_{k-l}^{-1}\left(\begin{array}{c}
y_{k-l} \\
\vdots \\
y_{k}
\end{array}\right)-\phi_{l} L_{k-l}^{-1} M_{k-l}\left(\begin{array}{c}
u_{k-l} \\
\vdots \\
u_{k+n-2-l}
\end{array}\right)
$$


$+\left(\begin{array}{cccc}\phi_{l-1} B_{k-l} & \ldots & \phi_{1} B_{k-2} & \phi_{0} B_{k-1}\end{array}\right)\left(\begin{array}{c}u_{k-l} \\ \vdots \\ u_{k+n-2-l}\end{array}\right)$

we obtain:

$$
Z_{k}=\phi_{l} L_{k-l}^{-1} Y_{k-l}-\left[\phi_{l} L_{k-l}^{-1} M_{k-l}-\Psi\right] U_{k-l}
$$

Supposing that $l=n-1$, the expression of $Z_{k}$ is then given as follows:

$$
\begin{gathered}
Z_{k}=\phi_{(n-1)} L_{k-(n-1)}^{-1} Y_{k-(n-1)} \\
-\left[\phi_{l} L_{k-(n-1)}^{-1} M_{k-(n-1)}-\Psi\right] U_{k-(n-1)}
\end{gathered}
$$

with:

$\Psi=\left[\begin{array}{lllll}\phi_{(n-1)-1} B_{k-(n-1)} & \ldots & \phi_{1} B_{k-2} & \ldots & \phi_{0} B_{k-1}\end{array}\right]$

\section{2-DOF controller using flatness based control}

By replacing $Z_{k}$ by its expression of (36) in the expression of the flatness-based control law given by (16).

The flatness-based control can be then written in the following form:

$$
S\left(k, q^{-1}\right) u_{k}=K(q) z_{k}^{d}-R(k, q) y_{k}
$$

with:

$$
S\left(k, q^{-1}\right)=\left(1+\Lambda_{k}\left[\phi_{n-1} L_{k-n-1}^{-1} M_{k-n-1}-\Psi\right]\right) \Pi^{*}
$$

and:

$$
R(k, q)=\Lambda_{k} \phi_{l} L_{k-n-1}^{-1} \Pi
$$

where:

$$
\Pi=\left(\begin{array}{lll}
q^{-n+1} & \ldots & 1
\end{array}\right)^{T}
$$

and:

$$
\Pi^{*}=\left(\begin{array}{lll}
q^{-n+1} & \ldots & q^{-1}
\end{array}\right)^{T}
$$

The final form of the controller allows us to obtain a 2DOF controller in LTV framework without need to define any observation dynamics and without resolution of the diophantine equation.

\section{ILlustrative Numerical EXAMPLE}

\section{A. Considered model}

Let's consider the following discrete-time system defining by the matrices:

$$
\begin{gathered}
A_{k}=\left[\begin{array}{ll}
0 & e^{-k T} \\
1 & e^{-k T}
\end{array}\right] \\
B_{k}=\left[\begin{array}{c}
1 \\
e^{-(k+1) T}
\end{array}\right], \quad C_{k}=\left[\begin{array}{ll}
0 & 1
\end{array}\right]
\end{gathered}
$$

This system is an academic second order model, with single input and single output used to highlight the effectiveness of the discrete-time flatness-based control approach in LTV SISO case. Noting that, $k$ is the iteration rank and $T=0.5 \mathrm{~s}$ is the simple time.

The open loop step response is given by Fig. 2 .

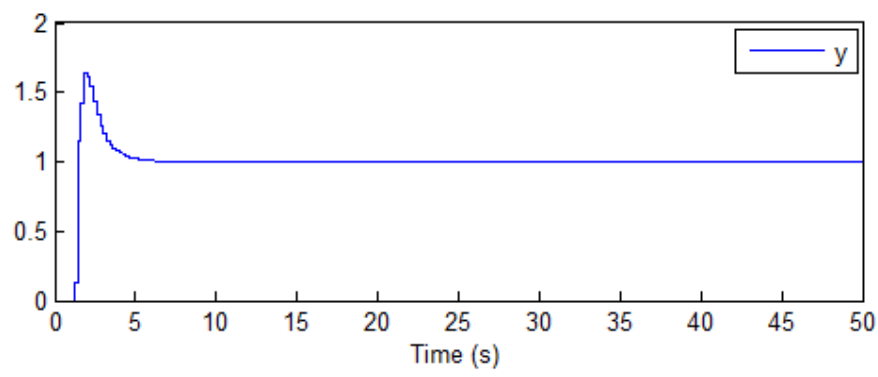

Fig. 2. Control law design.

\section{B. Desired Trajectory}

To achieve the implementation of this control law, firstly the definition of the trajectory is needed to be tracked by the considered system.

The aim is to force any system to track the given trajectory, so it's necessary to choose the reference trajectory, then we can determine correctly the endogenous parameters satisfying this objective with a correct dynamics. Let's consider the desired flat output defined as follows:

$z_{d}(k T)=z_{i n}+\left(21\left(\frac{k T}{50}\right)^{5}-35\left(\frac{k T}{50}\right)^{6}+15\left(\frac{k T}{50}\right)^{7}\right)$

$$
\times\left(z_{f i n}-z_{i n}\right)
$$

$z_{i n}$ and $z_{\text {fin }}$ are the initial and final values of the flat output that is determined endogenously from the initial and final values of the system outputs. The trajectory presented in the discrete-time is used as a reference for the flatness-based control using dead-beat observer.

The discrete-time desired trajectory is represented in Fig. 3.

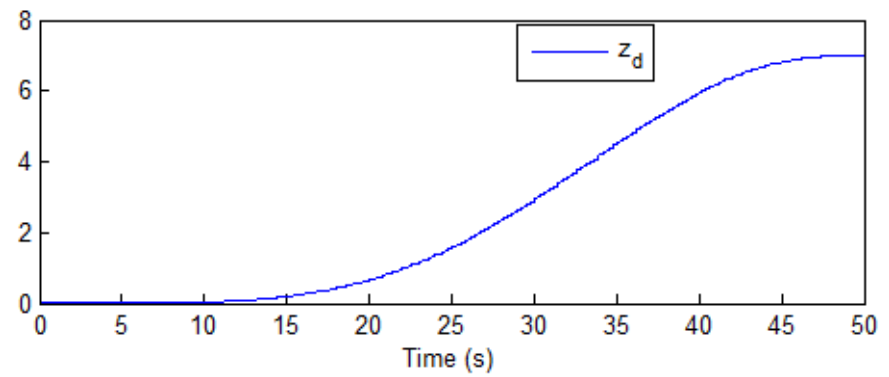

Fig. 3. Desired trajectory for the discrete-time flat output. 
C. Control design

From (14), the control law is given by:

$$
\begin{gathered}
u_{k}=z_{k+2}^{d}+k_{1} z_{k+1}^{d}+k_{0} z_{k}^{d}+\left(\gamma_{0}(k)-k_{0}\right) z_{k} \\
+\left(\gamma_{1}(k)-k_{1}\right) z_{k+1}
\end{gathered}
$$

After applying the controllable canonical form, the system matrices are given as follows:

$$
\begin{gathered}
\bar{A}_{k}=\left[\begin{array}{cc}
0 & 1 \\
-\gamma_{0}(k) & -\gamma_{1}(k)
\end{array}\right] \\
\bar{B}_{k}=\left[\begin{array}{c}
1 \\
e^{-(k+1) T}
\end{array}\right], \quad \bar{C}_{k}=\left[\begin{array}{ll}
0 & 1
\end{array}\right]
\end{gathered}
$$

with:

$$
\begin{gathered}
\gamma_{0}(k)=\frac{e^{(k+3)}-e^{(3)}+e^{(3 k+1)}}{e^{(2 k+1)}+e^{(4 k+1)}-e^{k}} \\
\gamma_{1}(k)=\frac{f_{1}+f_{2}}{f_{3}}
\end{gathered}
$$

and:

$$
\Lambda_{k}=\left[\begin{array}{c}
\frac{f_{4}}{f_{5}} \\
\frac{f_{6}}{f_{7}}
\end{array}\right]
$$

where:

$$
\begin{gathered}
f_{1}=e^{3} \times e^{k}-e^{1}-e^{2 k} \times e^{1} \\
f_{2}=e^{2 k} \times e^{4}+e^{3 k} \times e^{3} \\
f_{3}=e^{2 k} \times e^{2}-e^{k}+e^{4 k} \times e^{4} \\
f_{4}=100\left(e^{k+3}-e^{3}+e^{3 k+1}\right)+7\left(e^{2 k+1}+e^{4 k+1}-e^{k}\right) \\
f_{5}=100\left(e^{2 k+1}+e^{4 k+1}-e^{k}\right) \\
f_{6}=\frac{e^{k+4}+e^{3}+e^{2 k+3}}{e^{k+2}+e^{3 k+4}-1}-e^{k+1}\left(e^{-2 k}-1\right) \\
f_{7}=e^{k+2}+e^{3 k+4}-2.65
\end{gathered}
$$

The transition matrix is:

$$
\phi_{1}=\bar{A}_{k-1}, \quad \phi_{0}=1
$$

and:

$$
\Psi=\left[\begin{array}{ll}
\bar{A}_{k-1} \bar{B}_{k-2} & \bar{B}_{k-1}
\end{array}\right]
$$

As noted previously, this control law can lead to a $2 \mathrm{DOF}$ controller with the following parameters:

$$
\begin{gathered}
S\left(k, q^{-1}\right)=\left(1+\Lambda\left[\phi_{1} L_{k-1}^{-1} M_{k-1}-\Psi\right]\right) \Pi^{*} \\
R(k, q)=\Lambda \phi_{1} L_{k-1}^{-1} \Pi
\end{gathered}
$$

with:

$$
\begin{gathered}
S\left(k, q^{-1}\right)=\left[1-\left(\frac{s_{1} \times s_{2}}{s_{3}}\right)-\frac{s_{4}}{s_{5}}\right] q^{-1} \\
R(k, q)=\left[r_{1}, r_{2}\right] \times\left[\begin{array}{c}
q^{-1} \\
1
\end{array}\right]
\end{gathered}
$$

where:

$$
\begin{gathered}
s_{1}=\frac{e^{k+1} \cdot\left(e^{2 k+1}+1\right)-\left(e^{k+4}+e^{k+1}+20\right)}{e^{k+2}+e^{3 k+4}-1}+0.6 \\
s_{2}=e^{2 k}-e^{k+3}+e^{2}+e^{2 k+1}-e^{3 k+1}-e^{2 k+3}+e^{4 k+1}-e^{k} \\
s_{3}=e^{2 k+1}+e^{4 k+1}-e^{k} \\
s_{4}=100\left(e^{k+3}+e^{3 k+1}\right)+13\left(e^{2 k+1}+e^{4 k+1}-e^{k}\right)-2 \\
s_{5}=100\left(e^{2 k+1}+e^{4 k+1}-e^{k}\right)
\end{gathered}
$$

and:

$$
\begin{gathered}
r_{1}=\frac{e^{k+3}+0.13 e^{2 k+1}+e^{3 k+1}+0.13 e^{4 k+1}+0.13 e^{k}-20}{e^{2 k+1}+e^{4 k+1}-e^{k}} \\
r_{2}=\frac{\left(r_{3} \times r_{4} \times r_{5}\right)}{r_{6}} \\
r_{3}=e^{3 k+1}\left(e^{3 k+2}+e^{k}-1\right) \\
r_{4}=e^{k+5}-e^{6}+e^{3 k+1} \\
r_{5}=\frac{e^{k+1}\left(e^{-2 k}+1\right)-e^{k+4}+e^{2 k+3}+20}{e^{k+2}+e^{3 k+4}-1}+0.6 \\
r_{6}=\left(e^{5 k}-e^{2 k+5}+e^{3 k+4}\right)\left(e^{4 k}-e^{k+2}+e^{2 k+2}\right)
\end{gathered}
$$

A 2-DOF controller in LTV framework is obtained without defining any observation dynamics, the effectiveness of this control method is shown in the next section.

\section{Simulation Results}

After applying the control law signal shown in Fig. 4 to the considered system, the effectiveness of this method is proven and the system output follows the desired flat output with an error which tends to zero as shown in Fig. 6. Both the system output and the desired flat output are represented in Fig. 5.

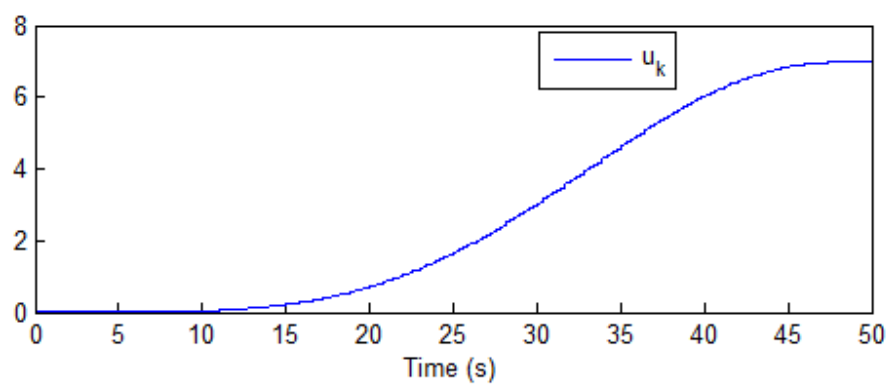

Fig. 4. The control signal $u_{k}$. 


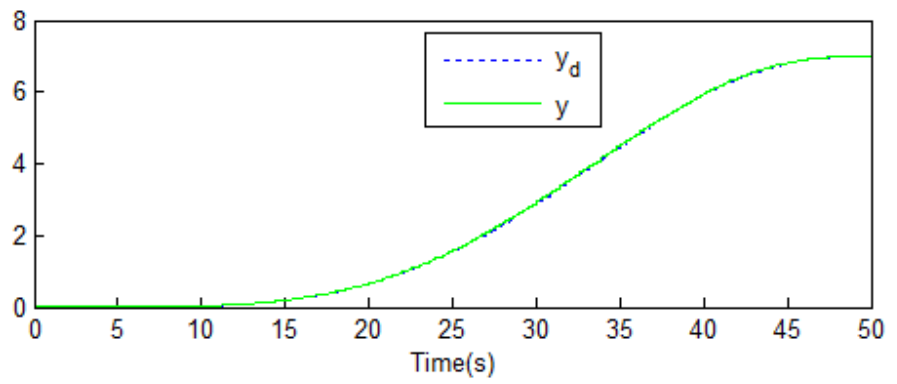

Fig. 5. System output and desired flat output: $y$ and $y_{d}$.

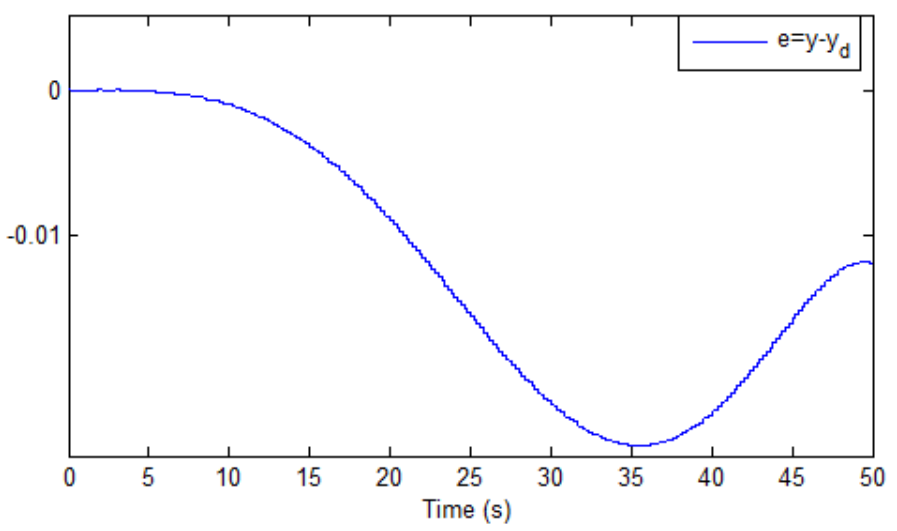

Fig. 6. Tracking error for the system output.

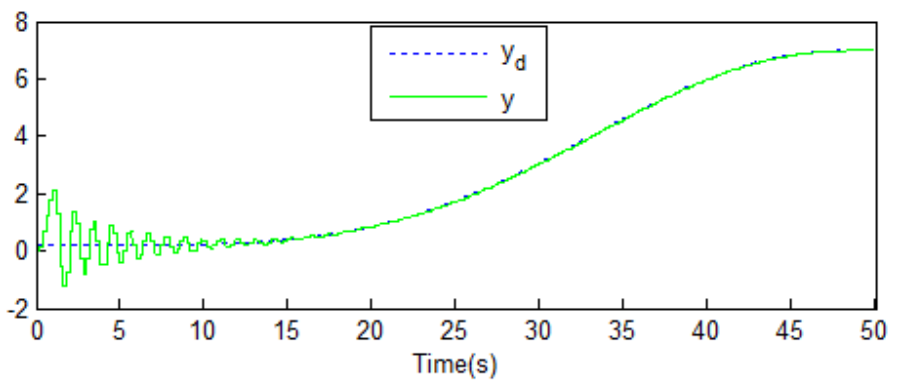

Fig. 7. System output and desired flat output: $y$ and $y_{d}$ with the first initial condition $\delta y_{0}=0.2$.

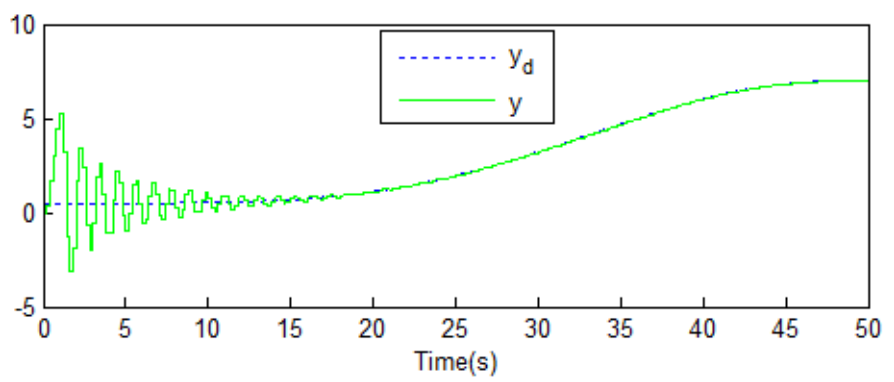

Fig. 8. System output and desired flat output: $y$ and $y_{d}$ with the second initial condition $\delta y_{0}=0.5$.

When applying two different initial conditions value to the desired trajectory, we obtain the results shown in Fig. 7 and 8

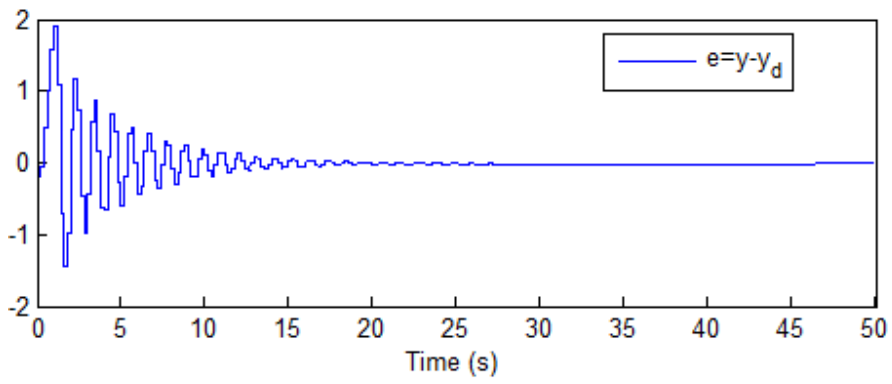

Fig. 9. Tracking error for the second initial condition.

with error for the first one given by Fig. 9 .

The linearization around a given trajectory applied to a nonlinear system leads to a linear time-varying system. The real time simulation of such method poses a very important field of study because of the strong symbolic calculations leading to a large size of polynomial matrices. Moreover, The robustness of such method touching the time-varying perturbations, the varying parameters and the practical implementation are prospects which will be carried out in the future works.

\section{CONCLUSION}

In this paper, a discrete-time control law using the flatness property with trajectory tracking is proposed. Many works which have used the fatness theory to achieve a trajectory tracking by solving bezout equation and carrying out a pole placement. Several difficulties gone through during the design of those methods. Contrary, with a quite few calculations and avoiding the above-mentioned problems our developed approach seems more effective. The design of this control law coupled with a dead-beat observer leads to a 2DOF controller without need to solve diophantine equation. Such a controller based on flatness implies the use of a dead-beat observer, leading to a direct interpretation of the closed loop poles, which are naturally constituted by the poles of the the tracking polynomial $K(q)$. This control law method shows good performances in terms of trajectory tracking in the case of LTV discrete-time systems.

\section{APPENDIX}

The matrix $\bar{T}_{k}$ is called the transformation matrix, its the tool that transforms any system to its controllable form [12]. In the LTV discrete-time case this matrix changes over time at each sample until convergence. The plant (1) becomes:

$$
\left\{\begin{array}{c}
Z_{k+1}=\bar{T}_{k+1} A_{k} \bar{T}_{k}^{-1} Z_{k}+\bar{T}_{k+1} B_{k} U_{k} \\
y_{k}=C_{k} \bar{T}_{k}^{-1} Z_{k}
\end{array}\right.
$$

with $\bar{A}_{k}=\bar{T}_{k+1} A_{k} \bar{T}_{k}^{-1}, \bar{B}_{k}=\bar{T}_{k+1} B_{k}$ and $\bar{C}_{k}=C_{k} \bar{T}_{k}^{-1}$ In the following, we give the steps to obtain the controllable canonical form. Consider the following vector sequence for $i=1 \ldots n$.

$$
\left\{\begin{array}{c}
\mathcal{R}_{0}=B_{k}, \quad i=0 \\
\mathcal{R}_{i}=A_{k} \mathcal{R}_{i-1}(k+1), \quad i=1 \ldots n
\end{array}\right.
$$


the controllability matrix is then written as:

$$
\mathcal{R}(k)=\left(\begin{array}{lll}
\mathcal{R}_{0}(k) & \ldots & \mathcal{R}_{n-1}(k)
\end{array}\right)
$$

if $\operatorname{rank}(R(k))=n$ and to be uniformly N-step controllable means that, for all $k$, the system is controllable on the interval $[k-N+1, \quad K+1]$. Then the effect of this variable change results in the following equation [12]:

$$
\overline{\mathcal{R}}_{i}(k)=\bar{T}_{k+1} \mathcal{R}_{i}(k)
$$

then:

$$
\mathcal{R}_{c}(k)=\bar{T}_{k+1} \mathcal{R}(k)
$$

When the pair $\left(\bar{A}_{k}, \bar{B}_{k}\right)$ is deduced by the variables change of the pair $\left(A_{k}, B_{k}\right)$, and if $\mathcal{R}(k)$ is non-singular, then the following form is obtained [7]:

$$
\bar{T}_{k+1}=\mathcal{R}_{c}(k) \mathcal{R}^{T}(k)\left(\mathcal{R}(k) \mathcal{R}^{T}(k)\right)^{-1}
$$

otherwise we have the following form:

$$
\bar{T}_{k+1}=\mathcal{R}(k) \mathcal{R}_{c}^{-1}(k)
$$

The condition of singularity of the matrix $\mathcal{R}_{k}$ is a controllability criterion in the discrete-time case. This property is preserved by the variables change [12]. The algorithm for constructing the controllable form is then:

- $\quad$ Construction of $\gamma(k)$ vector

$$
\gamma(k)=-\mathcal{R}^{-1}(k) \mathcal{R}_{n}(k)=\left(\begin{array}{lll}
\gamma_{0}(k) & \ldots & \gamma_{n-1}(k)
\end{array}\right)^{T}
$$

- Transformation $\bar{T}_{k+1}$ then $\bar{T}_{k}$ which puts the pair $\left(\bar{A}_{k}, \bar{B}_{k}\right)$ as in (5), (6) and (7).

\section{REFERENCES}

[1] Utkin, V.I., Variable structure systems wth sliding mode, IEEE Transaction on Automatic control, vol. 22, n. 2, pp.212-222, 1977.

[2] Carrol, J.J., Schneider, M., and Dawson, D.M., Integrator backstepping techniques for the traking control of permanent magnet bruch Dc motors, In conference Record of the 1993 IEEE Industry Applications Society Annual Meeting, pp.663-671, 1993.

[3] Passino, K.M. and Yurkovich, S., Fuyzzy control. Addison-wesley Longman. INC, 1998.
[4] Rotella, F., Carrillo, F. J. and Ayadi, M., Polynomial controller design based on flatness, Kybernetika, vol. 38, no. 5, pp. 571-584, 2002.

[5] Ben Abdallah, M., Ayadi, M., Rotella, F. et Benrejeb, M., Régulateurs polynomiaux par platitude pour la commande des systèmes non stationnaires, Conférence Internationale Francophone de l'Automatique, CIFA 2012, Grenoble.

[6] Ayadi, M., Contributions à la commande des systèmes linèaires plats de dimension finie, Thèse de Doctorat, Institut National Polytechnique de Toulouse, Tarbes 2002

[7] Rotella, F., Carrillo, F. and Ayadi, M., Digital flatness-based robust controller applied to a thermal process, IEEE International Conference on Control applications, Mexico, pp.936-941, 2001.

[8] Ben Abdallah, M., Ayadi, M. et Benrejeb, M., Flatness-based control of MIMO linear Systems, Transaction on Systems, Signals and Devices, vol. 6, n. 1, pp.1-24, 2011.

[9] Sleimi, M., Ben Abdallah, M. and Ayadi, M., Digital flatnessbased control design for LTI MIMO systems, IEEE 4th International Conference on control Engineering and Information Technology, CEIT, Hammamet, 2016.

[10] Benabdallah, M., Sur la commande par platitude de systèmes dynamiques SISO et MIMO, Thèse de Doctorat en Génie Electrique, Ecole Nationale d'Ingénieur de Tunis, 2014.

[11] Fliess, M., Levine, J., Martin, P. and Rouchon, P., Flatness and defect of non-linear systems: introductory and examples, International Journal of Control, vol. 61, no. 6, pp.1327-1361, 1995.

[12] Kamen, E. W., Fundamentals of linear time-varying systems, The control handbook, Control System Advanced Methods, second edition, Taylor and Francis Group, New York.

[13] Malrait, F., Martin, Ph. and Rouchon, P., Dynamic feedback transformations of controllable linear time-varying systems, nonlinear Control in the year 2000. Edite par Isodori, A., Lamnabhi- Lagarrigue, F., Respondek, W., Lecture Notes in Control and Information Sciences, vol. 259, pp. 55-62, Springer, London, 2001.

14] Bingulac, S. and Meadaws, H.E., Controllability ans observability in time-variable linear systems, SIMA Journal of control and optimization, no. 5, pp.64-73, 1967.

[15] Sun, H., ButT, S.S. and Asherman, H., Discrete-time flatness-based control for a twin rotor helicopter with an extended kalman ilter, IEEE International Conference on Advanced Intelligent Mechatronics, Banff, AB, Canada, 2016.

[16] Paulo, S. and Rouchon, P., Flatnessbased control of a single qubit gate, IEEE transactions on Automatic control, vol. 53, pp.775-779, 2008.

[17] HUANG, R., Output feedback tracking control of nonlinear time-varying systems by trajectory linearisation, Phd thesis, the Russ College of Engineering and Technology of Ohio University, 1967.

[18] Fiacchini, M. and Millerioux, G., Dead-Beat functional observers for discrete-time LPV systems with unknown inputs, IEEE transactions on Automatic control, vol. 58, pp.3230-3235, 2013. 